\title{
Treat-to-target strategies in pulmonary arterial hypertension: the importance of using multiple goals
}

\author{
O. Sitbon* and N. Galiè ${ }^{\#}$
}

ABSTRACT: Major advances have occurred in the treatment of pulmonary arterial hypertension (PAH) over the past decade. The advent of PAH-specific pharmacological treatments has offered hope to patients with a debilitating, progressive disease and a poor prognosis. Combined drug treatment offers improved benefits over monotherapy, and current treatment guidelines for PAH recommend a sequential add-on approach to combination therapy for patients in New York Heart Association (NYHA)/World Health Organization functional class (WHO FC) II-IV.

Goal-oriented therapy determines the timing of treatment escalation by inadequate response to known prognostic indicators. Close monitoring of patients aids the early identification of inadequate response, so that treatment can be escalated promptly and before the patient's condition deteriorates further. Existing treatment goals are based on baseline values of prognostic indicators, but it is vital to identify risk factors that are both relevant during treatment and that can be assessed during follow-up appointments. Data from different PAH aetiologies indicate that NYHA/WHO FC is the most appropriate prognostic marker, with 6-min walk distance and several haemodynamic parameters representing alternatives.

Future refinement of goal-oriented therapy could include the use of multiple prognostic markers, while additional, large clinical trials will answer questions concerning choice and combination of treatment goals.

KEYWORDS: Early intervention, New York Heart Association/World Health Organization functional class, prognosis, pulmonary arterial hypertension, treat to target

$\mathbf{T}$ he management of patients with pulmonary arterial hypertension (PAH) has advanced rapidly over the last decade or so, despite $\mathrm{PAH}$ therapy being a relatively new field of research. Increased research into the condition, and the introduction of $\mathrm{PAH}$-specific treatments, has led to a markedly improved prognosis and outcome for patients with this disease, as well as a better understanding of the pathological mechanisms behind it. Such advances are especially encouraging, considering that the first PAH-specific therapy was approved in 1995 and the first oral therapy was approved less than 10 yrs ago. There is still considerable room for improvement, although the estimated survival for patients diagnosed with $\mathrm{PAH}$ in the past decade has undoubtedly improved, according to historical data from the early 1990s [1, 2], some patients still have a very poor prognosis and a remarkably rapid deterioration in their condition [3]. Although novel therapies with different mechanisms of action are being developed and tested, the current focus is on improving patient outcomes using existing therapies. Primarily, the method employed is early diagnosis of PAH followed by treatment with first-line monotherapy [4, 5], but there is also increasing emphasis on the importance of escalating therapy if a patient does not respond to initial treatment.

Historically, the decision of whether or not to escalate treatment was largely based on the presence of clinical deterioration. If a patient's exercise capacity or haemodynamic measurements deteriorated, or if their functional class worsened, treatment escalation was considered [6-8]. Although escalation of treatment was also considered if parameters did not improve from their baseline values, no firm guidance was provided on which parameters were the most

\section{AFFILIATIONS}

*Hôpital Antoine Béclère, Université Paris-Sud 11, INSERM U999,

Clamart, France.

\#University of Bologna, Bologna, Italy.

\section{CORRESPONDENCE}

0 . Sitbon

Service de Pneumologie

Hôpital Antoine Béclère

92141

Clamart

France

E-mail: olivier.sitbon@abc.aphp.fr

Received:

Aug 262010

Accepted after revision:

Oct 112010

PROVENANCE

Publication of this peer-reviewed article was supported by Actelion Pharmaceuticals Ltd, Switzerland (unrestricted grant European Respiratory Review issue 118). 
important or which values to use as thresholds. Recently, efforts have focused on establishing an approach that allows for intervention before obvious clinical and functional worsening is observed, since waiting for deterioration is not an acceptable approach in a rapidly progressing disease like PAH. This kind of "treat-to-target" approach has been recommended by recent treatment guidelines [4].

Combination therapy, using two or more classes of drugs simultaneously, is one way of escalating treatment, and has been used successfully in the treatment of systemic hypertension and heart failure $[9,10]$. It is also an attractive option for the management of $\mathrm{PAH}$ because three separate signalling pathways known to be involved in the disease are targeted by present therapies: 1) the prostacyclin pathway; 2) the endothelin pathway; and 3 ) the nitric oxide (NO) pathway. Prostacyclin signalling is known to play a role in inducing pulmonary vasodilation and the inhibition of smooth muscle cell growth [11]. It is targeted by epoprostenol, the first drug to be approved for the treatment of $\mathrm{PAH}$, and by two other prostacyclin analogues, iloprost and treprostinil. Circulating endothelin is known to cause proliferation, hypertrophy, fibrosis, inflammation and vasoconstriction in patients with PAH [12]. The first oral therapy approved for treatment of PAH was bosentan, a dual endothelin-receptor antagonist, which was followed by the selective endothelin-receptor-A antagonists, ambrisentan and sitaxentan. The NO pathway is enhanced by the phosphodiesterase type- 5 inhibitors sildenafil and tadalafil, and is known to be involved in pulmonary vasodilation and inhibition of proliferation smooth muscle cells [13].

Randomised clinical trials over the past decade have demonstrated that monotherapy with these advanced treatments is associated with improved survival [14-17], increased exercise capacity, better haemodynamics [14, 18-24] and delayed time to clinical worsening $[18,19,21,25]$. Extensive real-world experience of these treatments has also accumulated over this time, together with improved knowledge of how to use these therapies to effectively manage PAH. For example, $>80,000$ people worldwide have been prescribed bosentan, and there are extensive post-marketing surveillance programmes in place for bosentan [26] and ambrisentan. Although some earlier, small trials of combination therapy may have been inconclusive $[27,28]$, the volume of evidence supporting its use is increasing. Results from the first, large-scale trials investigating combination therapy [29], or that included considerable numbers of patients on PAH treatments at baseline [21], suggest that combination therapy is an effective management option.

There are three different ways that combination therapy could, theoretically, be applied to the management of PAH. These are a sequential add-on approach, first-line combination therapy and a step-down approach. The most recent treatment algorithm from the European Society of Cardiology/European Respiratory Society for $\mathrm{PAH}$ recommends a sequential approach to combination therapy [4]. According to these guidelines, patients should be started on monotherapy and followed up regularly. If an inadequate clinical response is observed on monotherapy, adding a further therapy can be considered. Starting patients on combination therapy, known as "initial combination therapy", is another potential approach. Despite the possibility that such aggressive treatment of $\mathrm{PAH}$ could prevent clinical deterioration, it should only be considered for treatment of the most severely affected patients in New York Heart Association (NYHA)/World Health Organization functional class (WHO FC) IV [4], as little supporting evidence exists. Similarly, although it may be possible to start patients on combination therapy and then step-down treatment to monotherapy, this is a controversial proposal, with very little data or expert experience to support it.

This review will focus on the definition of inadequate treatment response and the use of treatment goals and discuss the use of sequential combination therapy as described in the most recent guidelines. The possibility of using more detailed data from prospective studies and combining different parameters to create more robust treatment goals will also be considered.

\section{DEFINING INADEQUATE TREATMENT RESPONSE}

Ultimately, the objective of treatment in $\mathrm{PAH}$ is to reduce functional impairment and prolong life. Goal-oriented therapy, a treatment strategy that uses known prognostic indicators as treatment targets, meets this objective by facilitating early intervention and therapeutic escalation before patients deteriorate (fig. 1). The key difference between goal-oriented therapy and previous approaches is that patients who are stabilised, or even those who improve slightly, can still receive escalated therapy if goals are not met. For instance, a patient who improved slightly in terms of 6-min walk distance (6MWD) but who did not reach the required threshold could still be given intensified treatment. It also requires the use of a variety of different parameters; stabilisation in NYHA/WHO FC III is not an acceptable outcome, even if improvements are observed in other parameters.

In 2005, a goal-oriented treatment algorithm for PAH and its outcomes was described by HOEPER et al. [30] (fig. 2). According to this algorithm, patients were started on first-line bosentan monotherapy and three treatment goals were predefined at baseline. If patients met these goals in follow-up visits monotherapy was continued. If a goal was not met, additional therapy was added. After 3 yrs of follow-up, survival estimates were improved over historical controls ( $80 \%$ versus $63 \%$ survival at 3 yrs) (fig. 3 ).

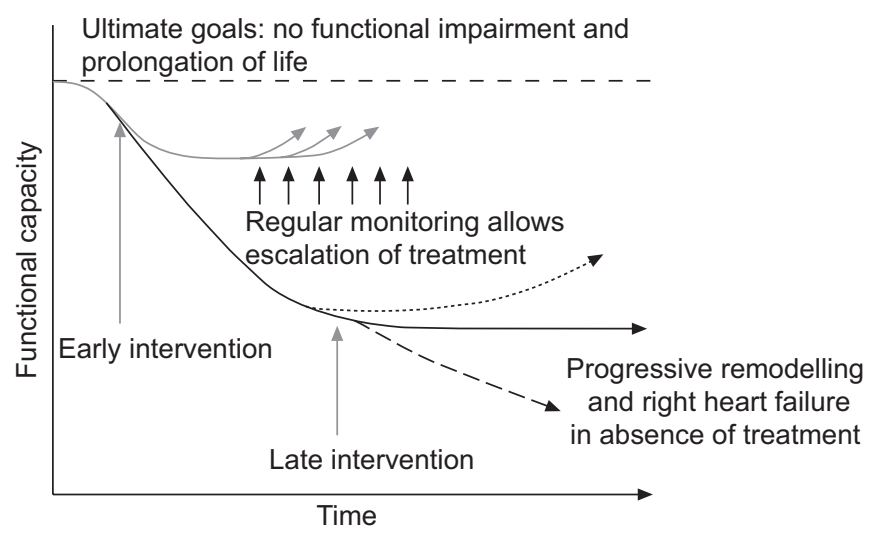

FIGURE 1. Schematic diagram showing the ideal approach to pulmonary arterial hypertension management, which involves regular monitoring and early intervention. 
Another study demonstrated a similar improvement in survival after using treatment goals to decide whether prostanoid therapy should be added to first-line bosentan [31].

Although goal-oriented treatment is a successful strategy, there is still much to learn about how best to apply our knowledge of risk and to identify which treatment goals are the most important. The next section will summarise risk stratification in PAH, and discuss how knowledge relating to risk factors can be improved.

\section{IDENTIFYING RISK FACTORS AND ESTABLISHING APPROPRIATE GOALS}

The key to choosing relevant treatment goals in a lifethreatening disease such as PAH is to identify parameters that correlate strongly with the risk of deterioration and mortality. Current treatment goals used in clinical trials, and those recommended by guidelines, are largely based on values of these parameters at baseline. Ideally, as goals will be used to guide decisions during patient follow-up visits, risk factors need to be identified that are relevant during treatment and that can be assessed during follow-up appointments.

\section{NYHA/WHO FC}

One of the most important factors to consider when setting treatment goals is functional capacity, as assessed by NYHA/ WHO FC. Not only is there is a large amount of congruent data available to support its use as a treatment goal, but the data relate to NYHA/WHO FC at baseline and during treatment. In addition, the supporting data are derived from a wide range of PAH aetiologies.

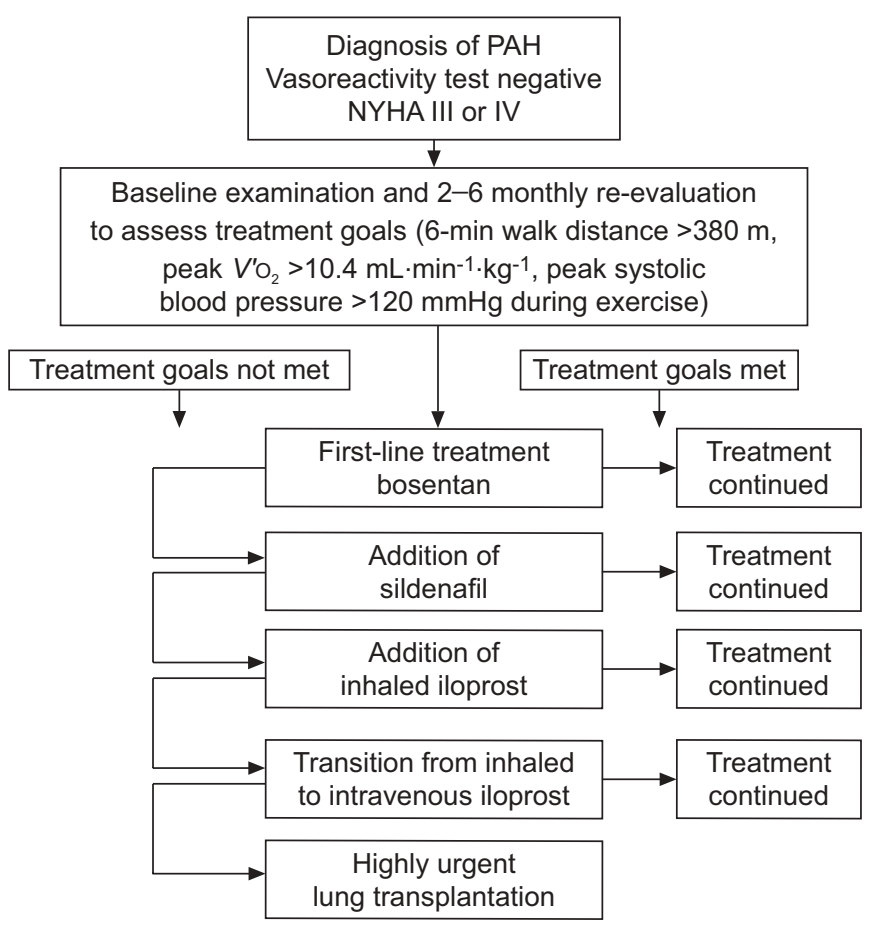

FIGURE 2. Therapeutic strategy for patients with pulmonary arterial hypertension (PAH). Goal-oriented therapy algorithm. NYHA: New York Heart Association; $\mathrm{V}^{\prime} \mathrm{O}_{2}$ : oxygen uptake. Reproduced from [30] with permission from the publisher.
Patients who are diagnosed in NYHA/WHO FC IV at baseline have a much worse prognosis compared to those who have less symptomatic PAH [2, 3, 15, 32]. In long-term studies of epoprostenol that enrolled patients in NYHA/WHO FC III and IV, survival estimates for patients in NYHA/WHO FC IV at baseline were much worse than those in NYHA/WHO FC III (47\% versus $71-81 \%$ at 3 yrs) $[15,32]$. Historical data collected before the advent of PAH-specific therapy [2] and the recent analysis of survival in a French cohort of PAH patients in the modern treatment era [3] also confirm the importance of NYHA/WHO at baseline.

The significance of improving to NYHA/WHO FC I or II during treatment with PAH-specific therapy is also highlighted in the available data. Patients with idiopathic PAH (IPAH) who improve to at least NYHA/WHO FC II following treatment with epoprostenol have a much better prognosis than those who do not improve (fig. 4) [15, 32]. Furthermore, NYHA/WHO FC after a period of treatment is prognostic in aetiologies other than IPAH. The survival rate for patients with Eisenmenger's syndrome was $32.2 \%$ for patients in NYHA/ WHO FC III/IV, compared with $14.1 \%$ for those in NYHA/ WHO FC I/II [33]. In a study of patients with PAH associated with systemic sclerosis (SSc), those who were in NYHA/WHO FC I/II after 4 months of treatment had survival rates of $100 \%$, $100 \%$ and $86 \%$ at 1, 2 and 3 yrs, respectively [34], contrasting with survival rates of $78 \%, 38 \%$ and $38 \%$, respectively, for those in NYHA/WHO FC III/IV. These data suggest that reaching at least NYHA/WHO FC II is a realistic treatment goal for all patients with $\mathrm{PAH}$, as it is consistently associated with improved survival prospects.

\section{Other parameters}

Baseline 6MWD is prognostic in IPAH patients [3], with three separate studies establishing prognostic thresholds of $332 \mathrm{~m}$, $250 \mathrm{~m}$ and $330 \mathrm{~m}$ [31, 32, 35]. Although absolute value for $6 \mathrm{MWD}$ reached on therapy is prognostic of survival [31, 32], relative change in $6 \mathrm{MWD}$ is not [32]. This is an important distinction: patients with severe $\mathrm{PAH}$ who start with a low $6 \mathrm{MWD}$ at baseline need to have a much greater improvement than those who have a better exercise capacity, if their prognosis is to improve.

Haemodynamic values at baseline that correlate strongly with prognosis include mean right atrial pressure $\left(\bar{P}_{\text {ra }}\right)$ and cardiac output/index [2, 3, 32], but not mean pulmonary arterial pressure $[3,32]$. Some parameters, such as $\bar{P}_{\mathrm{ra}}$, mixed venous oxygen saturation, increase in cardiac index and decrease in total pulmonary resistance, are also prognostic markers following treatment $[8,32]$. The level of pulmonary pressure is not associated with outcome in patients with $\mathrm{PAH}[3,32]$.

Data suggest that noninvasive measures, such as cardiopulmonary exercise testing (CPET) and echocardiography could be included as treatment goals. Peak oxygen uptake $\left(V^{\prime} \mathrm{O}_{2}\right.$,peak $)$ and peak systolic blood pressure at exercise are associated with long-term outcome [36], as are right atrial size, pericardial effusion, eccentricity index, Tei index and tricuspid annular plane systolic excursion [37-39]. Baseline levels of biomarkers, such as uric acid [40], brain natriuretic peptide (BNP) [41] and troponin $\mathrm{T}$ [42], are other possible prognostic factors. In one study, changes in plasma concentration of BNP at follow-up 


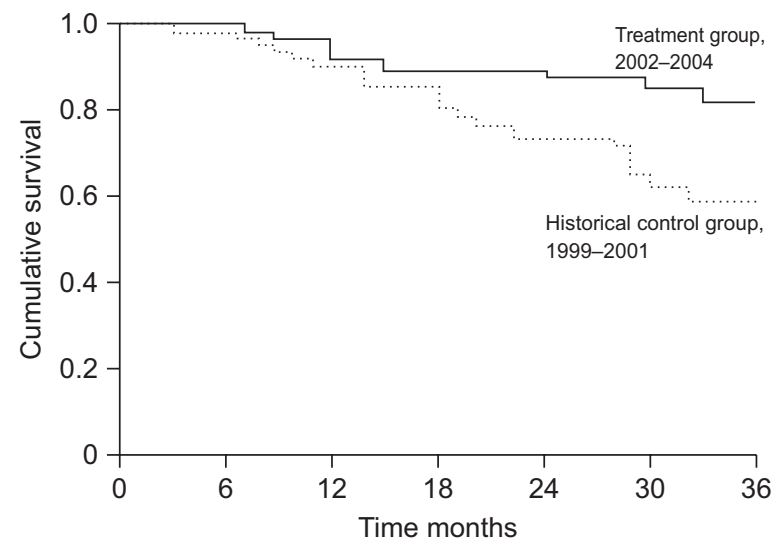

Subjects at risk $n$

$\begin{array}{lccccccc}\begin{array}{l}\text { Treatment } \\ \text { group }\end{array} & 89 & 83 & 69 & 61 & 46 & 43 & 37 \\ \begin{array}{l}\text { Historical } \\ \text { control group }\end{array} & 67 & 64 & 47 & 38 & 31 & 23 & 20\end{array}$

FIGURE 3. Kaplan-Meier estimates of survival in 89 patients with pulmonary arterial hypertension. Survival is improved by using goal-orientated therapy. Reproduced from [30] with permission from the publisher.

predicted outcome more accurately than baseline BNP values or haemodynamic parameters [41].

\section{The importance of using multiple goals}

The studies described previously have been used to inform choices of treatment goals in subsequent investigations and to influence PAH treatment guidelines. HOEPER et al. [30] set values for 6MWD (>380 m), $V^{\prime} \mathrm{O}_{2}$, peak $\left(>10.4 \mathrm{~mL} \cdot \mathrm{min}^{-1} \cdot \mathrm{kg}^{-1}\right)$ and peak systolic blood pressure at exercise $(>120 \mathrm{mmHg})$ to act as goals (fig. 2). PROVENCHER et al. [31] added prostanoid therapy if patients remained in NYHA/WHO FC III/IV and either 6MWD decreased by $10 \%$ or cardiac index was $<2.2 \mathrm{~L} \cdot \mathrm{min}^{-1} \cdot \mathrm{m}^{-2}$. As a result of the encouraging results observed in these studies, current joint guidelines from the European Society of Cardiology and the European Respiratory Society have endorsed this approach and recommend regular follow-up visits using a variety of known prognostic parameters (table 1) [4]. Although the latest recommendations from the 4th World Symposium on Pulmonary Hypertension are less detailed, they do highlight the importance of improving patients to, or maintaining them in, NYHA/WHO FC II [5].

Even the most robust treatment targets should not be taken in isolation, and experts recommend that a panel of treatment goals are used when assessing patients [4]. It may also be possible to improve prediction of survival and to increase the robustness of treatment goals by assessing the prognostic value of combined parameters through multidimensional risk assessment. For example, improvement to at least NYHA/ WHO FC II and normalisation of haemodynamic variables is strongly associated with better prognosis [17, 32], and prediction of survival is improved by combining two CPET measurements, compared with just one [36]. This approach is becoming increasingly ambitious, as demonstrated by the novel "risk calculator" statistical model developed using data from the REVEAL registry of patients with PAH [43]. Analysis

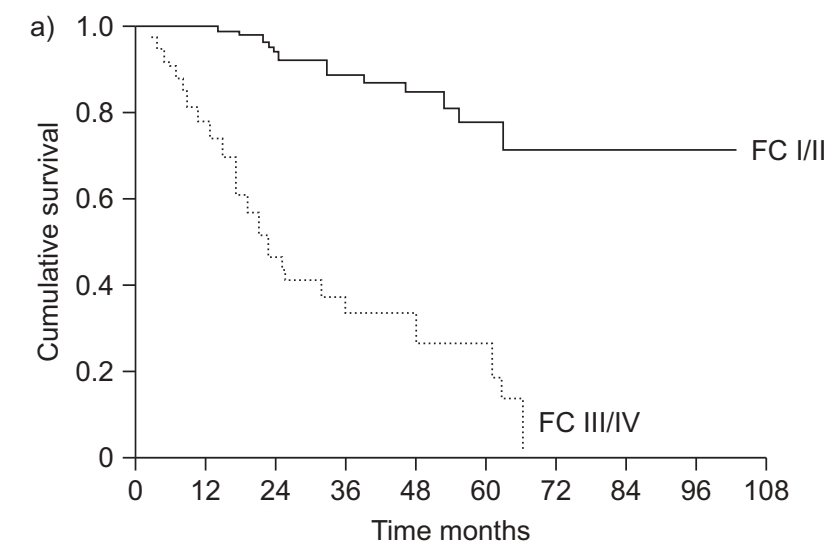

$\begin{array}{llllllllllll}\text { Subjects } & 91 & 84 & 69 & 48 & 32 & 17 & 7 & 3 & 1 & \text { FC I/II }\end{array}$

$\begin{array}{llllllllll}\text { at risk n } & 1 & 1 & 1 & 1 & 1 & 1 & & & \\ & 75 & 45 & 16 & 9 & 4 & 4 & & & \end{array}$

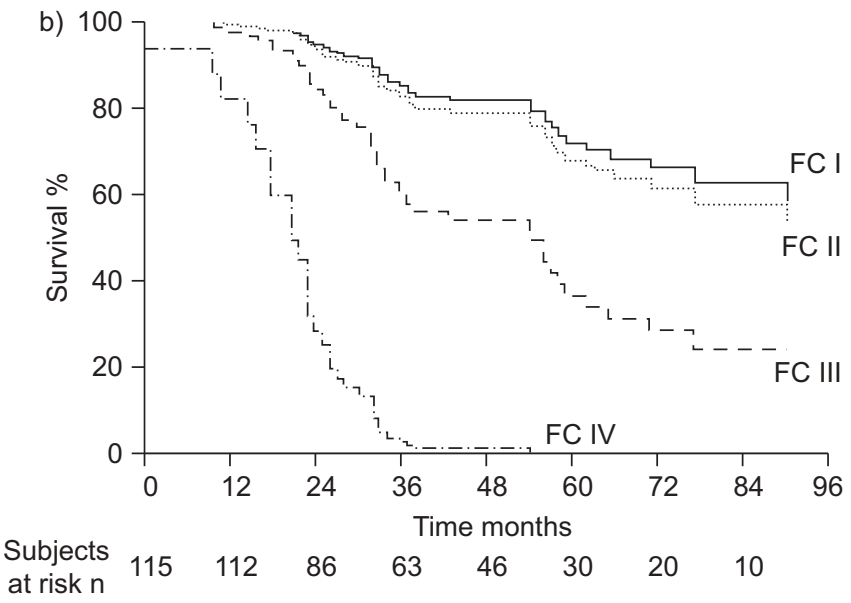

FIGURE 4. New York Heart Association/World Health Organization functional class (FC) at follow-up strongly predicts survival. a) Epoprostenol treatment for 3 months. Reproduced from [32] with permission from the publisher. b) Epoprostenol treatment for 1 yr. Reproduced from [15] with permission from the publisher.

of data from $>3,500$ patients was used to calculate prognostic values for the following parameters: $\mathrm{PAH}$ subgroup, demographics, NYHA/WHO FC, vital signs, 6MWD, BNP, echocardiography, pulmonary function tests and haemodynamic variables [44]. Newly diagnosed patients were then given a positive or negative value for each of these parameters, and the scores were added together to indicate whether they were at high risk of clinical worsening.

However, the vast majority of the results described previously come from retrospective studies that included both newly diagnosed patients (incidence cases) and those who were diagnosed prior to enrolment (prevalence cases). Despite the large patient population, even data from the REVEAL registry have their limitations because of the retrospective nature of any analyses that concern risk factors. In addition, the goals described above are primarily derived from patients with $\mathrm{IPAH}$ and PAH associated with SSc. Some goals, such as $6 \mathrm{MWD}$, may be more or less relevant for different patient groups. The exercise capacity and the rate of improvement for 


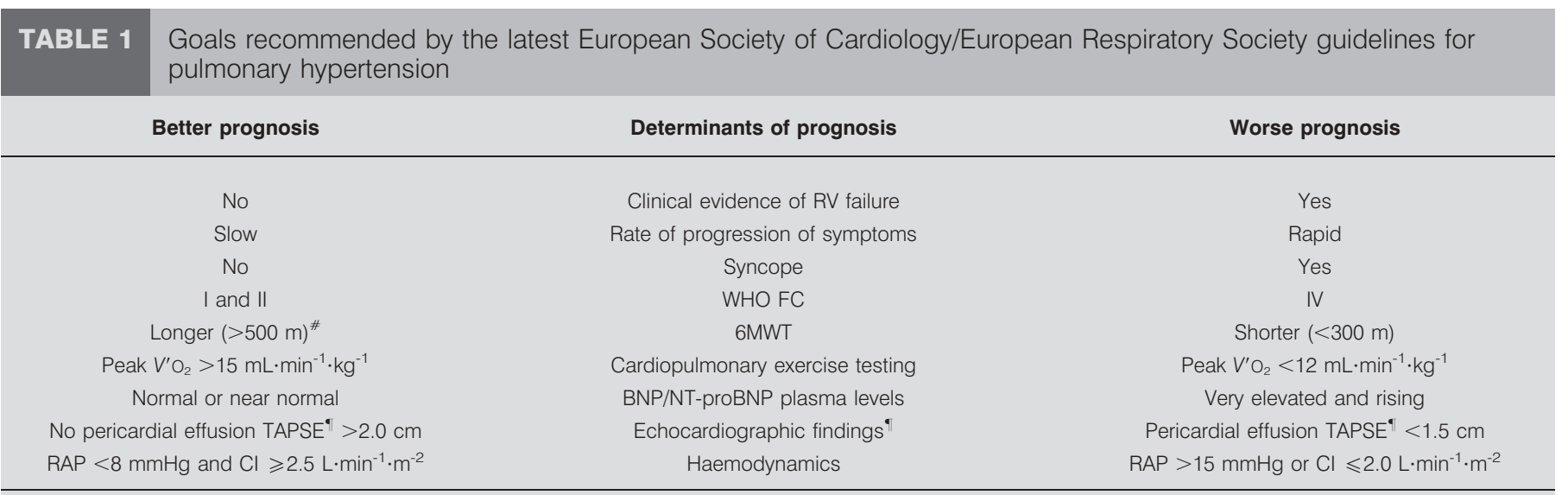

RV: right ventricle; WHO FC: World Health Organization functional class; 6MWT: 6-min walking test; $\mathrm{V}^{\prime} \mathrm{O}_{2}$ : oxygen uptake; BNP: brain natriuretic peptide; TAPSE: tricuspid annular plane systolic excursion; RAP: right arterial pressure; Cl: cardiac index. ${ }^{\#}$ : depending on age; ". TAPSE and pericardial effusion have been selected as they can be measured in the majority of patients. Reproduced from [4] with permission from the publisher.

IPAH patients is likely to differ from those for patients with PAH associated with connective tissue disease, who may have considerable comorbidities, and those with PAH associated with congenital heart disease.

\section{CHALLENGES FOR THE FUTURE}

Although the studies discussed previously provide valuable data that can aid the choice of relevant treatment goals, more information is required to improve the implementation of goaloriented therapy. It is still unclear which goals will provide the best outcomes for which patients, and which combinations of parameters are most appropriate for which subgroups of patients. It will also be important to properly investigate the difference in disease progression between incidence and prevalence cases. Further prospective studies with relatively large patient cohorts are required so that goals can be better adapted for individual patient needs.

An ongoing study in France (the EFORT study) may provide answers to some of the outstanding questions. Approximately 150-200 patients are expected to be enrolled in this prospective analysis of risk factors, creating a large cohort of exclusively incident patients. During a 3-yr follow-up period, a systematic assessment will be performed, using parameters such as NYHA/WHO FC, 6MWD, right heart catheterisation, echocardiography and biomarkers. According to the present guidelines, measurements will be taken at baseline, 3-4 months after treatment initiation and then once a year. This will help determine the most appropriate treatment goals by combining variables associated with the longest survival time. Importantly, this cohort will be limited to patients with IPAH, heritable $\mathrm{PAH}$ or anorexigen-associated $\mathrm{PAH}$, meaning that similar studies of PAH patients with different aetiologies will also be required.

There is also much that we need to learn about the effect of current treatment on the vasculature and disease progression. Although available evidence does suggest that early treatment can delay time to clinical worsening [25] and that goalorientated treatment does improve survival [30], data supporting this strategy is still relatively sparse. Results from clinical trials have demonstrated that PAH-specific drugs are strong vasodilators, resulting in improved haemodynamics [14, 18-24], and the three targeting pathways are associated with processes involved in disease progression in $\mathrm{PAH}$ [45]. However, there is no empirical evidence demonstrating disease modification as a direct effect of treatment. This could, theoretically, have an effect on the implementation of any treat-to-target strategy. For example, it might be argued that, as suggested by previous guidelines, it is best to wait for deterioration in chosen measurements rather than specify treatment goals [6]. This could allow the body to compensate on its own before having to resort to adding more treatment. Alternatively, early treatment that improves cardiac haemodynamics may protect the right heart during the progressive phase of vascular remodelling or even prevent malicious irreversible changes. Hopefully such questions can be answered by future research, such as an ongoing study which uses echocardiology to measure the extent of left and right ventricular remodelling before symptom onset and during pharmacological treatment in patients with PAH [46].

\section{CONCLUSION}

$\mathrm{PAH}$ is a progressive disease that requires regular monitoring and treatment escalation if treatment goals with initial monotherapy are not met. Currently, the best way to achieve treatment goals appears to be add-on combination therapy. At present, the data suggest that the clearest goal is improvement of patients to NYHA/WHO FC II, together with improvements in haemodynamic and functional parameters. Treatment goals have evolved considerably over the last decade and are becoming increasingly sophisticated, but more detailed data are required in order to identify the most relevant parameters. Targets need to become even more ambitious and robust in the future, both by using risk factors that are most relevant to patients on treatment and by combining these parameters.

\section{STATEMENT OF INTEREST}

O. Sitbon has relationships with drug companies including Actelion, Bayer Schering, GSK, Lilly, Pfizer and United Therapeutics, in addition to being an investigator in trials involving these companies relationships include consultancy service and membership of scientific 
advisory boards. He has received reimbursement for attending symposium and funds for research from Actelion, Pfizer, GSK, Lilly and Bayer Schering. He has received fees for speaking from Actelion, Bayer Schering, GSK, Lilly, Pfizer and United Therapeutics. N. Galiè reports having served on the advisory boards of Pfizer, Actelion, Schering, Encysive, Myogen, GSK, Eli Lilly and Mondobiotech and having been paid lecture fees by Actelion and Schering. He reports that his institute has received grant support from Pfizer, Actelion, Schering, Encysive, United Therapeutics, Eli-Lilly, Mondobiotec and Myogen.

\section{ACKNOWLEDGEMENTS}

We received editorial assistance from $T$. Newton at Elements Communications Ltd (Westerham, UK), supported by Actelion Pharmaceuticals Ltd (Allschwil, Switzerland).

\section{REFERENCES}

1 Thenappan T, Shah SJ, Rich S, et al. Survival in pulmonary arterial hypertension: a reappraisal of the NIH risk stratification equation. Eur Respir J 2010; 35: 1079-1087.

2 D'Alonzo GE, Barst RJ, Ayres SM, et al. Survival in patients with primary pulmonary hypertension. Results from a national prospective registry. Ann Intern Med 1991; 115: 343-349.

3 Humbert M, Sitbon O, Chaouat A, et al. Survival in patients with idiopathic, familial and, anorexigen-associated pulmonary arterial hypertension in the modern management era. Circulation 2010; 122: $156-163$.

4 Galiè N, Hoeper MM, Humbert M, et al. Guidelines for the diagnosis and treatment of pulmonary hypertension: The Task Force for the Diagnosis and Treatment of Pulmonary Hypertension of the European Society of Cardiology (ESC) and the European Respiratory Society (ERS), endorsed by the International Society of Heart and Lung Transplantation (ISHLT). Eur Heart J 2009; 30: 2493-2537.

5 Barst RJ, Gibbs JS, Ghofrani HA, et al. Updated evidence-based treatment algorithm in pulmonary arterial hypertension. J Am Coll Cardiol 2009; 54: Suppl. 1, S78-S84.

6 Galiè N, Torbicki A, Barst R, et al. Guidelines on diagnosis and treatment of pulmonary arterial hypertension: The Task Force on Diagnosis and Treatment of Pulmonary Arterial Hypertension of the European Society of Cardiology. Eur Heart J 2004; 25: 2243-2278.

7 Badesch DB, Abman SH, Ahearn GS, et al. Medical therapy for pulmonary arterial hypertension: ACCP evidence-based clinical practice guidelines. Chest 2004; 126: Suppl. 1, 35S-62S.

8 Badesch DB, Abman SH, Simonneau G, et al. Medical therapy for pulmonary arterial hypertension: updated ACCP evidence-based clinical practice guidelines. Chest 2007; 131: 1917-1928.

9 Frishman WH, Landau A, Cretkovic A. Combination drug therapy with calcium-channel blockers in the treatment of systemic hypertension. J Clin Pharmacol 1993; 33: 752-755.

10 Pitt B, Zannad F, Remme WJ, et al. The effect of spironolactone on morbidity and mortality in patients with severe heart failure. Randomized Aldactone Evaluation Study Investigators. N Engl J Med 1999; 341: 709-717.

11 Kataoka M, Nagaya N, Satoh T, et al. A long-acting prostacyclin agonist with thromboxane inhibitory activity for pulmonary hypertension. Am J Respir Crit Care Med 2005; 172: 1575-1580.

12 Giaid A, Yanagisawa M, Langleben D, et al. Expression of endothelin-1 in the lungs of patients with pulmonary hypertension. N Engl J Med 1993; 328: 1732-1739.

13 Mehta S. Sildenafil for pulmonary arterial hypertension: exciting, but protection required. Chest 2003; 123: 989-992.

14 Barst RJ, Rubin LJ, Long WA, et al. A comparison of continuous intravenous epoprostenol (prostacyclin) with conventional therapy for primary pulmonary hypertension. The Primary
Pulmonary Hypertension Study Group. N Engl J Med 1996; 334: 296-302.

15 McLaughlin VV, Shillington A, Rich S. Survival in primary pulmonary hypertension: the impact of epoprostenol therapy. Circulation 2002; 106: 1477-1482.

16 McLaughlin VV. Survival in patients with pulmonary arterial hypertension treated with first-line bosentan. Eur J Clin Invest 2006; 36: Suppl. 3, 10-15.

17 Sitbon O, McLaughlin VV, Badesch DB, et al. Survival in patients with class III idiopathic pulmonary arterial hypertension treated with first line oral bosentan compared with an historical cohort of patients started on intravenous epoprostenol. Thorax 2005; 60: 1025-1030.

18 Channick RN, Simonneau G, Sitbon O, et al. Effects of the dual endothelin-receptor antagonist bosentan in patients with pulmonary hypertension: a randomised placebo-controlled study. Lancet 2001; 358: 1119-1123.

19 Rubin LJ, Badesch DB, Barst RJ, et al. Bosentan therapy for pulmonary arterial hypertension. N Engl J Med 2002; 346: 896-903.

20 Galiè N, Olschewski H, Oudiz RJ, et al. Ambrisentan for the treatment of pulmonary arterial hypertension: results of the ambrisentan in pulmonary arterial hypertension, randomized, double-blind, placebo-controlled, multicenter, efficacy (ARIES) study 1 and 2. Circulation 2008; 117: 3010-3019.

21 Galiè N, Brundage BH, Ghofrani HA, et al. Tadalafil therapy for pulmonary arterial hypertension. Circulation 2009; 119: 2894-2903.

22 Galiè N, Ghofrani HA, Torbicki A, et al. Sildenafil citrate therapy for pulmonary arterial hypertension. $N$ Engl J Med 2005; 353: 2148-2157.

23 Barst RJ, Langleben D, Frost A, et al. Sitaxsentan therapy for pulmonary arterial hypertension. Am J Respir Crit Care Med 2004; 169: 441-447.

24 Barst RJ, Langleben D, Badesch D, et al. Treatment of pulmonary arterial hypertension with the selective endothelin-A receptor antagonist sitaxsentan. J Am Coll Cardiol 2006; 47: 2049-2056.

25 Galiè N, Rubin LJ, Hoeper MM, et al. Treatment of patients with mildly symptomatic pulmonary arterial hypertension with bosen$\tan$ (EARLY study): a double-blind, randomised controlled trial Lancet 2008; 371: 2093-2100.

26 Humbert M, Segal ES, Kiely DG, et al. Results of European postmarketing surveillance of bosentan in pulmonary hypertension. Eur Respir J 2007; 30: 338-344.

27 Humbert M, Barst RJ, Robbins IM, et al. Combination of bosentan with epoprostenol in pulmonary arterial hypertension: BREATHE-2. Eur Respir J 2004; 24: 353-359.

28 Hoeper MM, Leuchte H, Halank M, et al. Combining inhaled iloprost with bosentan in patients with idiopathic pulmonary arterial hypertension. Eur Respir J 2006; 28: 691-694.

29 Simonneau G, Rubin LJ, Galiè N, et al. Addition of sildenafil to long-term intravenous epoprostenol therapy in patients with pulmonary arterial hypertension: a randomized trial. Ann Intern Med 2008; 149: 521-530

30 Hoeper MM, Markevych I, Spiekerkoetter E, et al. Goal-oriented treatment and combination therapy for pulmonary arterial hypertension. Eur Respir J 2005; 26: 858-863.

31 Provencher S, Sitbon O, Humbert $\mathrm{M}$, et al. Long-term outcome with first-line bosentan therapy in idiopathic pulmonary arterial hypertension. Eur Heart J 2006; 27: 589-595.

32 Sitbon $\mathrm{O}$, Humbert $\mathrm{M}$, Nunes $\mathrm{H}$, et al. Long-term intravenous epoprostenol infusion in primary pulmonary hypertension: prognostic factors and survival. J Am Coll Cardiol 2002; 40: 780-788.

33 Dimopoulos K, Inuzuka R, Goletto $S$, et al. Improved survival among patients with Eisenmenger syndrome receiving advanced therapy for pulmonary arterial hypertension. Circulation 2010; 121: $20-25$.

34 Launay D, Sitbon O, Le Pavec J, et al. Long-term outcome of systemic sclerosis-associated pulmonary arterial hypertension 
treated with bosentan as first-line monotherapy followed or not by the addition of prostanoids or sildenafil. Rheumatology 2010; 49: 490-500.

35 Miyamoto S, Nagaya N, Satoh T, et al. Clinical correlates and prognostic significance of six-minute walk test in patients with primary pulmonary hypertension. Comparison with cardiopulmonary exercise testing. Am J Respir Crit Care Med 2000; 161: 487-492.

36 Wensel R, Opitz CF, Anker SD, et al. Assessment of survival in patients with primary pulmonary hypertension: importance of cardiopulmonary exercise testing. Circulation 2002; 106: 319-324.

37 Raymond RJ, Hinderliter AL, Willis PW, et al. Echocardiographic predictors of adverse outcomes in primary pulmonary hypertension. J Am Coll Cardiol 2002; 39: 1214-1219.

38 Yeo TC, Dujardin KS, Tei C, et al. Value of a Doppler-derived index combining systolic and diastolic time intervals in predicting outcome in primary pulmonary hypertension. Am J Cardiol 1998; 81: 1157-1161.

39 Forfia PR, Mathai SC, Fisher MR, et al. Hyponatremia predicts right heart failure and poor survival in pulmonary arterial hypertension. Am J Respir Crit Care Med 2008; 177: 1364-1369.

40 Nagaya N, Uematsu M, Satoh $T$, et al. Serum uric acid levels correlate with the severity and the mortality of primary pulmonary hypertension. Am J Respir Crit Care Med 1999; 160: 487-492.

41 Nagaya N, Nishikimi T, Uematsu M, et al. Plasma brain natriuretic peptide as a prognostic indicator in patients with primary pulmonary hypertension. Circulation 2000; 102: 865-870.

42 Torbicki A, Kurzyna M, Kuca P, et al. Detectable serum cardiac troponin $\mathrm{T}$ as a marker of poor prognosis among patients with chronic precapillary pulmonary hypertension. Circulation 2003; 108: 844-848.

43 Badesch DB, Raskob GE, Elliott CG, et al. Pulmonary arterial hypertension: baseline characteristics from the REVEAL Registry. Chest 2010; 137: 376-387.

44 Benza RL, Miller DP, Gomberg-Maitland M, et al. Predicting survival in pulmonary arterial hypertension: insights from the Registry to Evaluate Early and Long-Term Pulmonary Arterial Hypertension Disease Management (REVEAL). Circulation 2010; 122: $164-172$.

45 Humbert M, Sitbon O, Simonneau G. Treatment of pulmonary arterial hypertension. N Engl J Med 2004; 351: 1425-1436.

46 Badano LP, Ginghina C, Easaw J, et al. Right ventricle in pulmonary arterial hypertension: haemodynamics, structural changes, imaging, and proposal of a study protocol aimed to assess remodelling and treatment effects. Eur J Echocardiogr 2010; 11: 27-37. 